\title{
Entrelacs
}

Cinéma et audiovisuel

5 | 2005

La Machine

\section{Quand le cinéma transporte}

Alexandre Tylski

\section{OpenEdition}

\section{Journals}

Édition électronique

URL : http://journals.openedition.org/entrelacs/137

DOI : 10.4000/entrelacs. 137

ISSN : 2261-5482

Éditeur

Éditions Téraèdre

Édition imprimée

Date de publication : 1 novembre 2005

Pagination : 92-96

ISBN : 978-2-912868-70-1

ISSN : $1266-7188$

Référence électronique

Alexandre Tylski, «Quand le cinéma transporte », Entrelacs [En ligne], 5 | 2005, mis en ligne le 01 août 2012, consulté le 20 avril 2019. URL : http://journals.openedition.org/entrelacs/137 ; DOI : 10.4000/ entrelacs.137

Ce document a été généré automatiquement le 20 avril 2019.

Tous droits réservés 


\title{
Quand le cinéma transporte
}

\author{
Alexandre Tylski
}

«On peut, par certains dispositifs de rythme, de rime et d'assonance, bercer notre imagination, la ramener du même au même en un balancement régulier... $»^{1}$

1 Un rituel s'engage, un billet vite déchiré. Tel serait un des pactes d'entrée du cinéma, une chose conçue pour être vite brisée. Nature éphémère du périple, on balise un temps, brièveté de toute nouveauté, inutilité de toute matérialité. Seuls l'esprit et l'imaginaire compteraient désormais? Notre identité, en tout cas, sera dorénavant ce billet balafré, nous n'existerons et ne serons acceptés en ce lieu qu'à la condition d'être, au préalable, déchirés.

2 Corps déchiqueté, contorsion cruelle, que l'aventure cicatrisera ou non, ce ticket ouvert, béant telle une gueule de fauve, autographe, est un peu la promesse d'une odyssée méritée, d'un passage vers d'autres mondes ; un décollage et un recollage.

3 Nous partons en voyage mais, à l'inverse des séjours organisés, nous partons sans bagage - si ce n'est notre propre corps, unique malle à souvenirs dans l'aventure à venir.

4 « ô Seigneur! Ouvrez-moi les portes de la nuit, afin que je m'en aille et que je disparaisse! " ${ }^{2}$. C'est une pérégrination et la croyance que nous puissions enfin effacer des maux de notre esprit. Dans l'entrebâillement timide, apparaît un immense champ moelleux de pousses pourpre où se blottissent les corps, et par-dessus lesquels les crânes se dépassent.

5 Ouvrir franchement la porte, souffle coupé, et dans la saisie originelle retrouver les impressions de Maupassant: "Quand on pénètre dans la chapelle, on demeure d'abord saisi comme en face d'une chose surprenante... $\|^{3}$

6 Puis, se trouver une place ici-bas, dans l'obscure alvéole : au milieu, ou sur le côté, comme dans le lit de notre chambre. Une place qui nous ressemble : devant, au feu, ou au fond, proche des battements de cœur du projecteur. Mais la meilleure place, pour reprendre Pagnol, n'est-ce pas celle où le réalisateur place la caméra? Salle d'attente, selon Topor, horizon d'attente. 
7 Certains s'extasient et rient avant le film, pour eux nulle religiosité, ils s'échauffent dans le plus terre à terre détachement, et profitent à juste raison de leur brève liberté de parole. Nous ne sommes alors pas loin des sportifs avant un match ou une course : «(...) ils séchauffent, prennent enfin place au départ. Eux et le starter jouent à se narguer. $\aleph^{4}$ Le starting-block que l'on fustige parfois insolemment du regard, c'est ici l'écran laiteux. Il nous domine tranquillement ce beau repère. Le duel commence.

8 Têtes courbées, nous sommes bloqués dans une conque de velours; douce régression et renaissance dans la matrice, dans le ventre de la baleine, "berceau de l'imagination ", " incubation $\gg .^{5}$ Dans l'immobilité retrouvée après un parcours du combattant, certains pensent alors au bruit du paquet de bonbon des enfants derrière, d'autres au cadre vert « sortie de secours ", d'autres encore rêvent au parfum de leur voisine, et, un peu au film, accessoirement. Mais, alors que ceux-là songent au film pour la première fois depuis fort longtemps, voilà que les lumières s'évanouissent. Et la lumière fuit.

9 Les lumières de la salle s'éteignent lentement sur tout, sur les chevelures, les étoffes, les mains, les lèvres. Un moment presque érotique. Là, quand les voix s'amenuisent, puis chuchotent, puis disparaissent, là quand le corps semble s'éclipser dans le silence et l'immobilité alors qu'il bouillonne plus que jamais. Là, quand rien d'autre n'existe sinon le subjectile magique, l'écran fenêtre, la toile qui fait naître. Là, quand le silence règne enfin. C'est un peu le premier fondu du film. On nous prie presque de fermer un instant les yeux ; invitation au rêve, crépuscule des yeux.

10 "Chez les Pueblo, on se raconte les mythes la nuit, et cela, dit-on, fait avancer les étoiles. ${ }^{6}$ Dans la chambre noire, où l'on se devine à peine, à l'image de l'expérience photographique, le corps est plongé dans le bain révélateur; dans ce là d'ombres et de profondeurs semblables à la couleur de la terre : «(...) la terre ouvre son sein fertile et prodigue ses trésors aux heureux peuples qui la cultivent pour eux-mêmes: elle semble sourire et s'animer au doux spectacle de la liberté ; elle aime à nourrir des hommes. ${ }^{7}$

11 La noirceur n'est cependant qu'apparence dans cette salle d'une obscure clarté. Outre ce monde d'idées qui s'ouvre à nous, le faisceau-mère, au-dessus, veille au grain. Luisant, fascinant, céleste pinceau dévoilant les poussières de notre vie, cet immense doigt pointé est le phare de notre nuit.

12 Là-haut, des roues décorent la cabine, on y dispose les bobines, déroule le ruban de rêve, puis la " gamelle $»^{8}$ l'avale. Les projectionnistes, conducteurs de trains ou de tracteurs. Le projecteur et ses rails intérieurs engloutissent sels d'argent, ester nitrique de cellulose à $10 \%$ d'azote additionné de camphre, gélatine, collodion, etc.

13 La pellicule, cette algue de l'imaginaire a tout de la feuille d'arbre, c'est une membrane en appel de lumière pour vivre et s'épanouir, c'est une peau (du latin, pellicula "petite peau »), délicate, féminine. Une longue langue que l'on introduit dans une carcasse pour que celle-ci s'exprime.

14 Car ce projecteur est parlant, criant de vérité même lorsqu'on est en sa présence. Si parlant qu'il fit dire aux premiers spectateurs la nature sonore et musicale, des premiers films prétendument «muets». Les rimes et les rythmes de cette machine sont en soi esthétiques et marquent déjà l'aurore de l'œuvre d'art.

15 «Un tracteur dans un garage, écrivait Gilbert Simondon, n'est qu'un objet technique (...) quand il est au labour, et sincline dans le sillon pendant que la terre se verse, il peut être perçu comme beau. Tout objet technique, mobile ou fixe, peut avoir son épiphanie esthétique, dans la mesure où il prolonge le monde et s'insère en lui. $»^{10}$ 
16 La «pelloche ", la mer, le "projo ", la terre; le soleil naîtra à la mise en route de l'appareil, au contact de deux épidermes électriques, tel que l'évoque Ingmar Bergman dès la première image de Persona (1961). Or, comme l'écrit Merger en incipit de son livre La Naissance: «La véritable naissance remonte donc plus haut (que l'accouchement), à l'instant où l'union de deux cellules crée la vie de l'homme. "

17 En bas, dans la salle, le visage des spectateurs s'illumine à nouveau dans l'interrogatoire perpétré par l'écran voyeuriste. L'odeur du projecteur envahit la cabine, cette même odeur d'ossature mécanique qui transmit au jeune Bergman sa vocation de cinéaste. Ce parfum ineffable qui prit racine dans le chrono-photographe, le kinétoscope, la lanterne magique, les ombres chinoises, le phénakistiscope, le phono-scope, le praxinoscope, le stroboscope, le zootrope. C'est une odeur de révolutions technologiques, une odeur de guerres. «(...) les projecteurs se remuaient sans cesse, flairant l'ennemi, le cernant de leurs lumières jusqu'au moment où les avions aiguillés bondiraient en chasse pour le saisir. ${ }^{11}$ Le phare écrasé contre le canevas annonce le début du corps à corps entre la toile lactée et les assiégés. Il les avertit d'une prochaine disparition, la leur, peut-être.

Dans Nourritures terrestres, Gide écrivait: "Qu'aimes-tu tant dans les départs, Ménalque? Il répondit: l'avant-goût de la mort. " L'incipit de L'Incompris (1966) de Luigi Comencini a ce parfum-là. Entrée en matière pour le moins mortifère avec cette caméra fixée sur le véhicule en mouvement semblable à un aigle sur une épaule ou à la grande faucheuse. La voiture, noire, avance vers une vaste demeure. C'est la voiture d'un père angoissé à l'idée d'annoncer à son fils la mort de sa mère. Le fils en mourra. Ce travelling avant initial apporte littéralement la mort à la maison familiale au fond du plan. Vision matricielle « fixée » à ce corbillard allégorique, au commencement du film.

À peine né, né à peine, le film affronte déjà la fin. Cette mise au monde par l'appareillage est une poussée contre le mur, le vide et l'inconnu. Ce sera le cas avec la voiture noire dès l'ouverture de Huit et demi de Federico Felini (1963) ou de la roue de voiture dès l'image mère de Bleu de Krystof Kieslowski (1992). Et dans certains débuts de films, ce sera une moto qui ouvrira le récit (La Vie de Jésus de Bruno Dumont, 1994), ou un camion (Voyage au bout de l'enfer de Michael Cimino, 1978), ou un navire (Mort à Venise de Luchino Visconti, 1971), ou, fréquemment, un train.

20 La Bête Humaine (1938) de Jean Renoir nous plonge d'entrée dans le feu intérieur d'une locomotive et ses flammes furieuses (Renoir a su comme Zola personnifier le feu). Du cœur enflammé de la machine, la caméra recule et présente deux hommes au charbon (naturalisme) et le paysage sur les bords défiler dans un flou effrayant (impressionnisme). Dès l'incipit, un paysage flou, comme balafré par des langues de flammes embrasant le monde à l'entour. Cette esthétique dichotomique raconte la folie galopante du héros (Jean Gabin), meurtrier dans les tripes, devenu machine lui-même. Animalité vague, instable. Vitesse du monde « entée » sur l'individu, enfer d'une condition bien réelle.

21 En 1995, Dead Man ${ }^{12}$ de Jim Jarmusch débuta aussi par le train ; machine à vapeur noire et fantomatique laissant présager l'artillerie et le métal envahissant la nature - et les disparitions humaines qui s'en suivent. «Le fruit, dès ses premiers jours, porte en lui le principe de sa pourriture. »13 Dans L'An 01 (1972), c'est moins la mort que le «train-train » anesthésiant de la gare que Jacques Doillon met en avant dès la première image du film, avec l'attente des usagers sur le quai et l'arrivée du train. La scène est filmée de manière diamétralement opposée à celle des frères Lumière (cf. l'arrivée en gare de la Ciotat, 1895). Filmé par Doillon de l'autre côté du quai, et non sur le quai lui-même, le plan est 
une mise en abyme de la machine cinéma elle-même, réflexivité sur un dispositif originel. Ici le zoom avant va contre le plan fixe des frères Lumière, le zoom avant comme le corps de la caméra qui se serait développé depuis sa naissance. La machine s'est mutée, transfigurée, vivante. Une révolution est en marche.

Train et cinéma sont liés par les dates, et par nature. Et c'est souvent au commencement même d'un film que cette réunion a lieu, avec la confirmation d'une même origine, d'un même point de départ - ce "point de départ " ayant pour objet premier le départ, le voyage. Qu'il soit létal ou libérateur, le voyage unit le cinéma au train. Ils transfigurent le paysage quotidien, le remuent, l'ouvrent, le saignent, et déchirent l'âme, la transportent, la transcendent. Pagnol engageait Regain (1937) avec un plan de train à vive allure, regain ainsi engagé. ${ }^{14} \mathrm{Et}$ le film se bouclait dans les sillons, en rails, d'un champ. «Terre, n'est-ce pas que tu veux, en nous, renaitre?", demande Rilke.

Plus que l'idée de la mort, c'est peut-être aussi et surtout la matrice qui palpite au cœur de la machine à voyager dans les temps. Poussée en avant, poussée du ventre matriciel, poussée depuis la salle obscure vers la lumière de l'écran. On pense à des débuts de films fulgurants où le film lance autant la machine que la machine propulse le film dans le corps des spectateurs. Ainsi Un Homme est passé de John Sturges (1955) avec ce train fonçant ex abrupto dans une musique tonitruante, ou le train entrant très vite dans un tunnel dès l'ouverture de La Cité des Femmes (1980) de Fellini.

Cette vitesse machinique dès l'aurore d'un film se trouve aussi dans la première image du pré-générique de Danger: Diabolik (1967) de Mario Bava : des motards roulant en trombe. Un plan coupé très vite, puis raccordé dans l'axe, sous l'effet de la table de montage. ${ }^{15}$ Une ouverture comme un moyen de transport. Bava réitère ensuite cette célérité dans le fragment-mère, là aussi très fugace, juste après le générique introductif, dévoilant un bateau lancé à toute vitesse. Le film sera une débauche de fulgurance, de machines en tout genre, une course-poursuite autant entre les forces de l'ordre et le voleur hérö̈que du film, qu'entre le film et nous. La machine au commencement même du dispositif cinématographique, au commencement même des films et de leur récit. Machines infernales, grosses machines hollywoodiennes ou petites machines filmiques, films sur les machines ou films de machinistes, tous les films ont pour cœur, pivot, la machine.

"(...) qu'est-ce que la machine? On peut, on doit, séparer nettement la machine de l'outil. Il ne semble pas, cependant, que cette discrimination fondamentale soit admise aujourd'hui. On parle couramment de machine à écrire, de machine à calculer, alors qu'il y a entre ces deux appareils une différence essentielle : la première est un outil, la seconde une véritable machine. $»^{16}$

Nous pouvons être tentés de voir le cinéma avant tout comme un outil ; outil pédagogique pour les uns, outil de propagande et de contre-propagande pour les autres. Outil de recherche, outil de débroussaillage, ou outil inutile mais indispensable, aussi. Mais le cinéma reste l'invention en marche et fils de la "marchine », celle qui élève l'Homme audessus des bêtes et le renvoie parfois à sa bestialité, lieu mouvant de matérialités et de transcendances.

Aujourd'hui, la place des ordinateurs dans l'art du cinéma n'a pas nécessairement transformé l'outil-cinéma en machine sans âme ou sans amour. Les films restent marqués par la même lèvre, balafre, originelle. Cette palpitation généreuse engagée par «la machine ronde » laissant la pensée changer de sens, ce grand ouvrage de l'Esprit qui nous porte, la Terre. 
Nota Bene: le présent texte a été une première fois effacé et perdu par l'automutilation d'un ordinateur. L'article a été réécrit de manière différente puis, à nouveau, perdu et écrasé par un second crash quelques semaines plus tard. Les machines auront finalement choisi d'épargner la troisième version, présentée ici. Une version peut-être plus élogieuse envers les machines que les deux autres, le saurons-nous jamais?

\section{NOTES}

1. Henri Bergson, in Le Rire, Ed. PUF, 1958, p. 62.

2. Victor Hugo, in Les Contemplations. Ed. Nelson, IV, XIII.

3. Guy de Maupassant, in La Vie errante. Ed. Louis Conard, 1909, la Sicile.

4. Jean Prévost, in Plaisirs des sports. Ed. Gallimard, 1925, p. 105.

5. Termes repris à Paul Nizon in Genèses du roman..., " Trouver un ton ", Ed. CNRS 1993, p. 210.

6. Marcel Mauss, in Le Manuel d'ethno- graphie, Ed. Payot, 1989. P. 120.

7. Jean-Jacques Rousseau, in Julie, Ed. Garnier 1935, IV, XVII.

8. Ancien terme désignant le projecteur.

9. Expression empruntée à Etienne Ithurria.

10. Gilbert Simon-don, in Du mode d'existence des objets techniques. Ed. Aubier, 1958. p. 185.

11. Marcel Proust, in Le Temps retrouvé. Ed. Gallimard, 1927, t. III, p. 759.

12. La ville dans le film de Jarmusch s'appelle Machine et sera aussi la cause des morts à venir.

13. Ernest Renan, in L'Avenir de la science, Ed. Gallimard, 1947., t. III, p. 1085.

14. L'immersion forte et inaugurale dans les films n'est pas l'apanage des films dits postmodernes comme il l'a été écrit ici et là, mais parcourt bel et bien l'histoire du cinéma de sa naissance à nos jours ; et pour longtemps encore si l'on reconnaît la nature fulgurante de l'art cinématographique.

15. Le montage, machine à voyager dans les temps dont Petro Costa étudiera les rouages dans Où gît votre sourire enfoui ?,2003).

16. Daniel-Rops, Le Monde sans âme, p. 67.

\section{AUTEUR}

\section{ALEXANDRE TYLSKI}

Allocataire-Moniteur au Lara / ESAV. Doctorant en Cinéma. Rédacteur en Chef de la revue en ligne Cadrage.net 